Supporting Information

\title{
Regulation Mechanisms of Carbon Dots in the Development of Lettuce and Tomato
}

Erfeng Kou, ${ }^{a, b}$ Yuying Yao ${ }^{a}$, Xian Yang ${ }^{b}$, Shiwei Song, ${ }^{b}$ Wei Li, ${ }^{a}$ Yunyan Kang, ${ }^{b}$ Songnan $Q u,{ }^{\mathrm{c}}$ Riyue Dong, ${ }^{a, b}$ Xiaoqin Pan, ${ }^{a, b}$ Dongna Li, ${ }^{a}$ Haoran Zhang, ${ }^{a}$ Bingfu Lei, ${ }^{*}{ }^{a, b, d}$

${ }^{a}$ Key Laboratory for Biobased Materials and Energy of Ministry of Education, College of Materials and Energy, South China Agricultural University, Guangzhou 510642, P. R. China

${ }^{\mathrm{b}}$ College of Horticulture, South China Agricultural University, Guangzhou 510642, P. R. China

${ }^{\mathrm{c}}$ Institute of Applied Physics and Materials Engineering, University of Macau, Taipa, Macau, P. R. China

d Maoming Branch, Guangdong Laboratory for Lingnan Modern Agriculture, Guangdong Maoming 525100, P. R. China

*Corresponding Author: Email: tleibf@scau.edu.cn.

Number of pages: 5

Number of figures: 7

Number of tables: 1 


\section{Characterizations of CDs}

High-resolution transmission electron microscopy (HRTEM) images were collected in a JEOL-2010 electron microscope. The Fourier transform infrared (FT-IR) spectra were measured by a Nicolet Avatar 360 FT-IR spectrophotometer. X-ray photoelectron spectroscopy (XPS) spectra were obtained by employing an X-ray photoelectron spectroscope (AXIS ULTRA DLD, Kratos). UV-Vis absorption spectra were taken on a JASCO V-570 spectrophotometer. Photoluminescence spectra were recorded with an F-7000 Hitachi fluorescence spectrofluorometer. Laser scanning microscopy (LSM) was performed with LSM 710, Zeiss. FLS980 Edinburgh Instrument spectrofluorometer measurement system was used for the measurement of the absolute quantum yield, and then the QY is determined by QY=Lsam / (ERef - Esam). Zeta potentials were determined by a Zeta potential analyzer (Brookhaven Instruments Corporation).

\section{Properties of CDs}

(a)

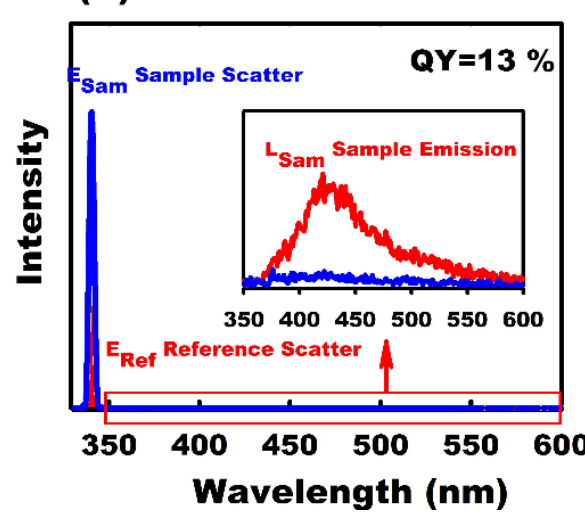

(b)

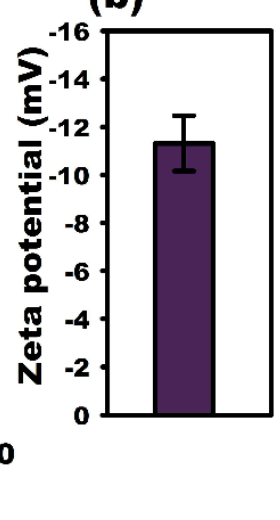

Figure S1. (a) The absolute quantum yield (QY) of CDs upon $340 \mathrm{~nm}$ excitation in water. (b) Zeta potential of CDs $(0.066 \mathrm{mg} / \mathrm{mL})$
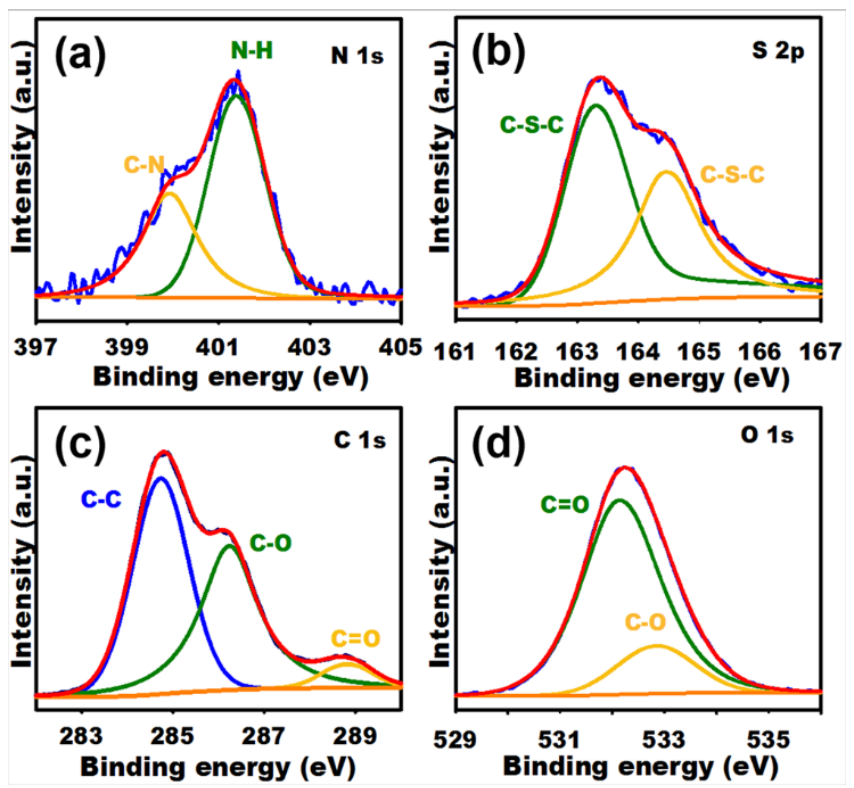

Figure S2. High resolution XPS spectra of C 1s (a), N 1s (b), O 1s (c), and S 2p (d). 


\section{The biocompatibility of CDs}

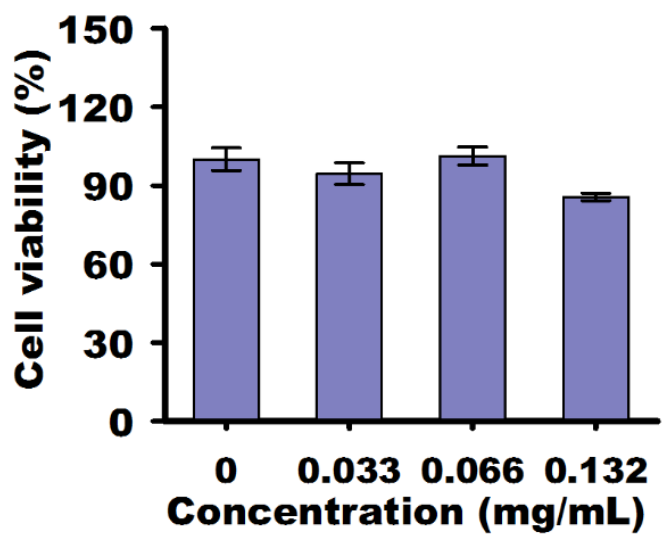

Figure S3. Viability of cells after $24 \mathrm{~h}$ treatment with different concentrations of CDs. Error bars indicate standard errors of four biological replicates.

The uptake of CDs in plants

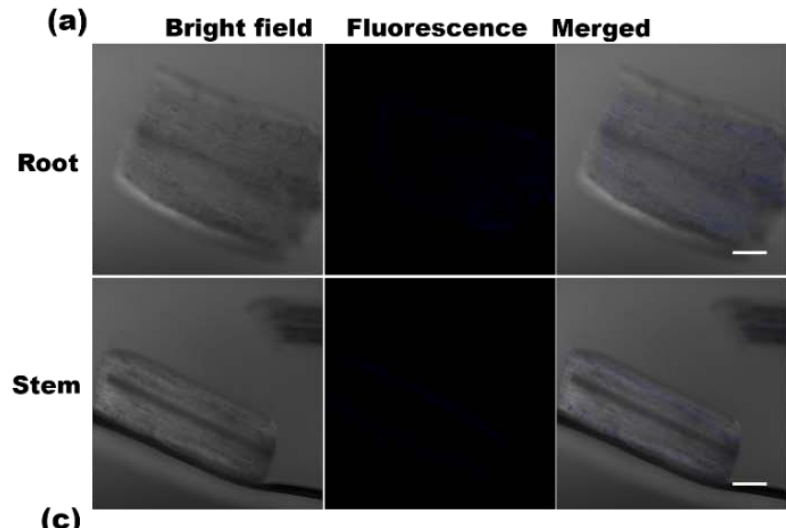

(b)
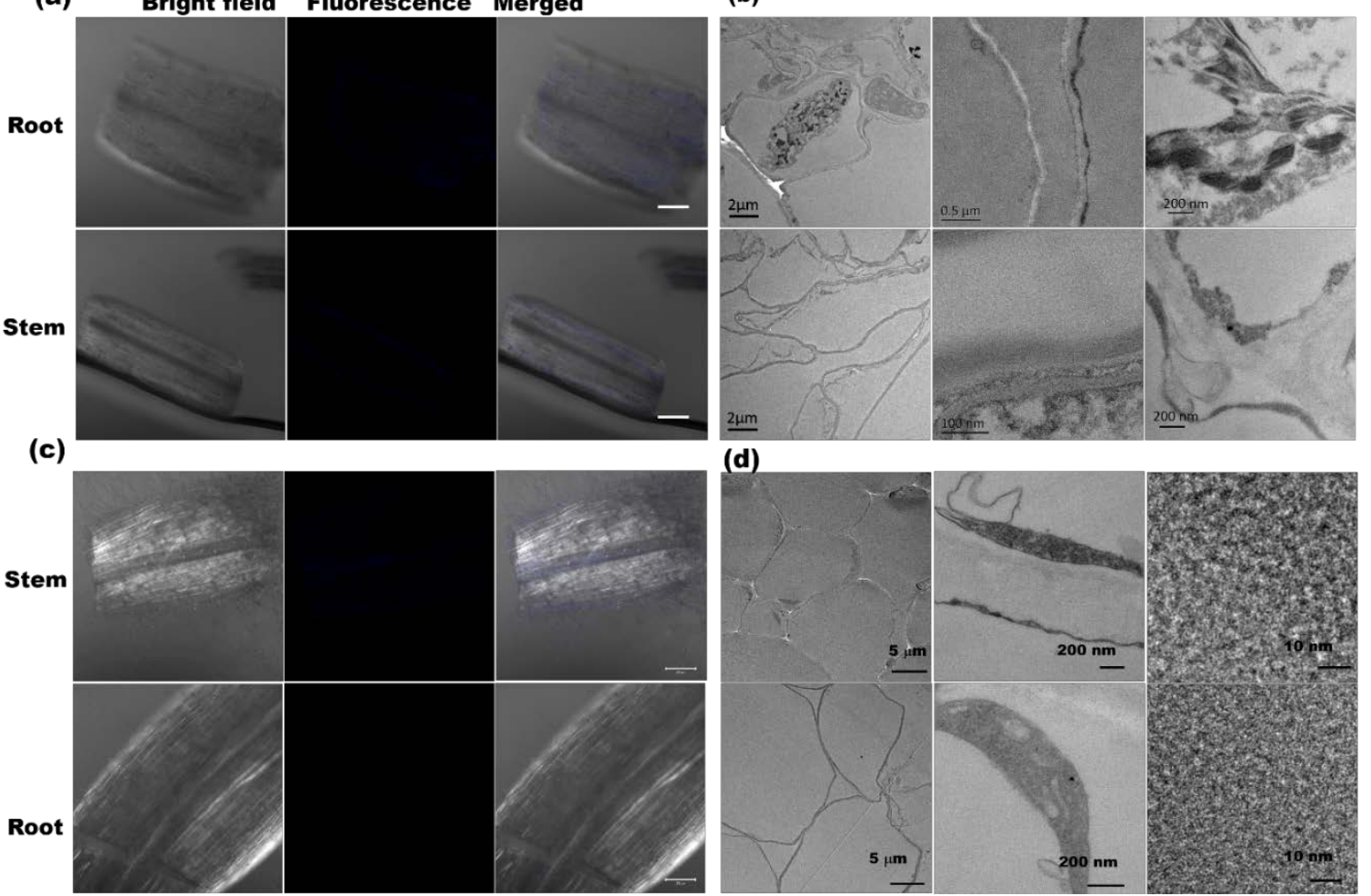

(d)

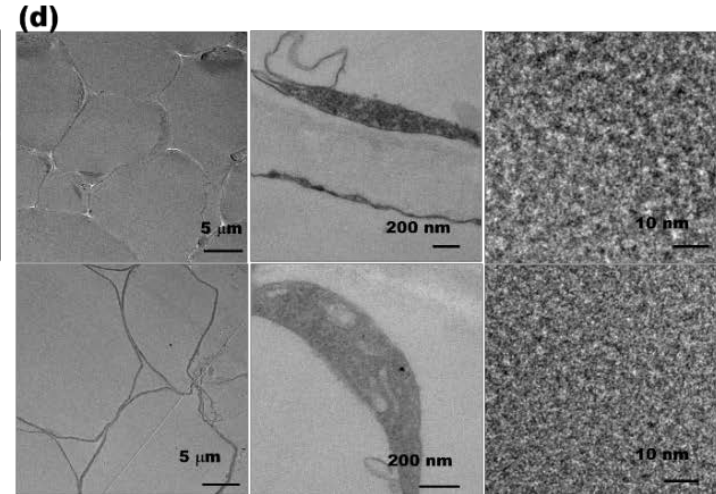

Figure S4. Penetration of CDs into plant cells. (a) and (c) are confocal images of stem and root of lettuce and tomato, respectively, in $1 \frac{1}{2}$ MS medium without CDs. (b) and (d) are HRTEM image for the root and stem of lettuce and tomato, respectively, cultured in of $1 / 2$ MS medium without CDs. 
CDs accelerates germination of seeds and elongation in seeding roots and hypocotyls

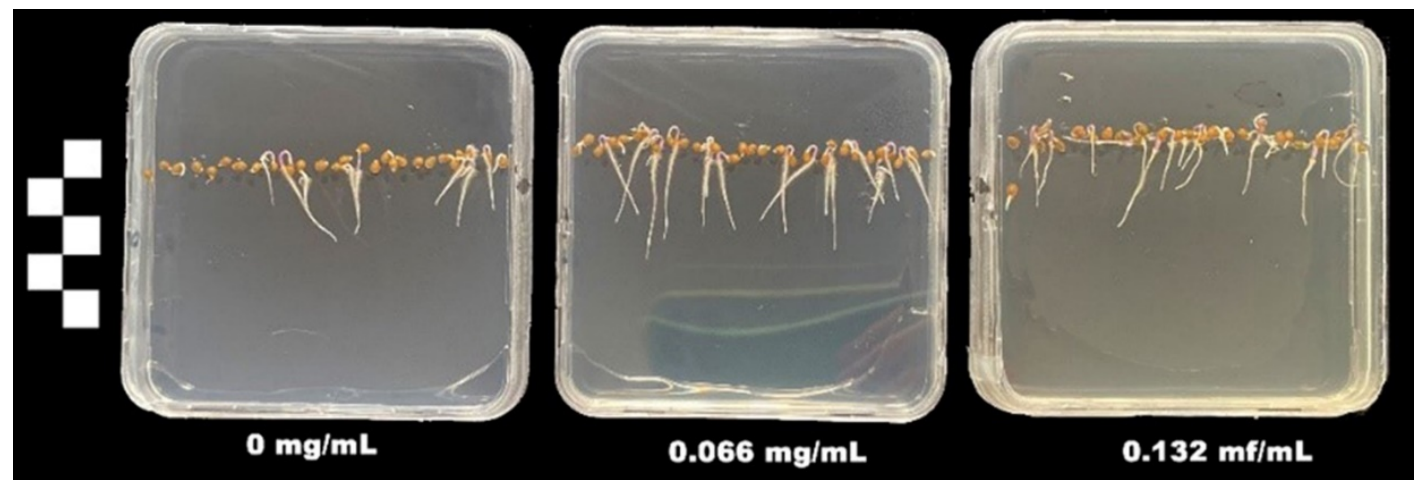

Figure S5. Tomato seeds germination after cultured four days in $1 \frac{1}{2}$ MS medium with and without CDs.

CDs enhanced the yield and nutritional quality in mature plants

(a)

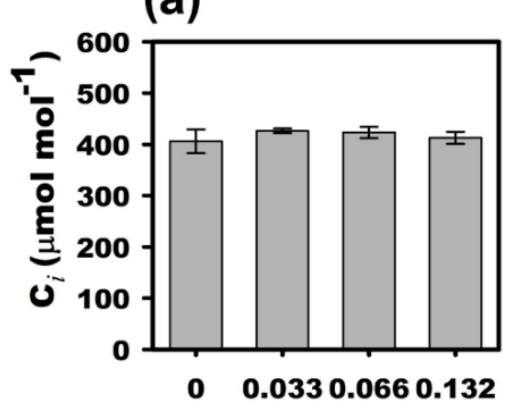

(b)

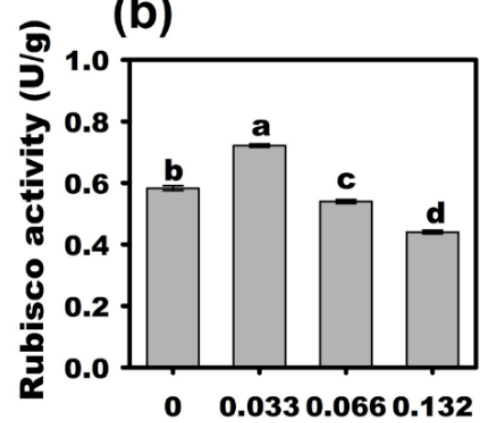

(c)

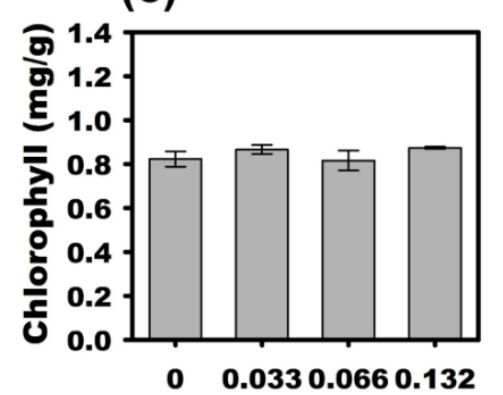

Figure S6. The intercellular $\mathrm{CO}_{2}$ concentration (Ci) and Rubisco activity (b) and the content of total Chlorophyll in mature lettuce leaves. Values with different letters indicate significant differences at $p<0.05$ according to Duncan's multiple-range tests. Error bars indicate standard errors of three biological replicates.

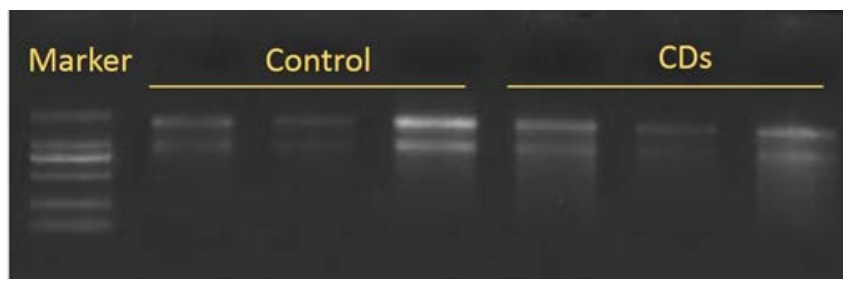

Figure S7. Electrophoretograms gel image of tomato seeding hypocotyl total RNA exposure to $1 / 2$ MS medium with and without CDs $(0.066 \mathrm{mg} / \mathrm{ml})$ after ten days duration of incubation. 
Table S1. Primer sequences

\begin{tabular}{ll}
\hline Primer name & Primer sequence (5’ $\left.\sim 3^{\prime}\right)$ \\
\hline SlAqp2-F & AGGATGTGAAGGTTGGAGCA \\
SlAqp2-R & GTGGTGCCTCCTTGTAGTCC \\
SlPIP2-1-F & GGAGCCATTTGTGGTTGTGG \\
SIPIP2-1-R & GGGTAGAGGCGCAAGTACAG \\
SIPIP2-5-F & TCCAGCACCACTTATTGACCC \\
SIPIP2-5-R & CACCAAAGGCCCAAGCAATG \\
SIPIP2-7-F & ACCGTTGCTCGATTTTGCTG \\
SIPIP2-7-R & TCATGCCTCCAAAAGCCCAA \\
SITIP2-1-F & ATTCGGATTGGCTCTCGGTG \\
SITIP2-1-R & CCATTCCTCCGGTCACAACT \\
SITIP2-3-F & GTCACTCTGGGATTGGCTGT \\
SITIP2-3-R & CACTCATCCCAGCAGCAACT \\
SIUBI3-F & CGTGAAAACCCTAACGGGGA \\
SlUBI3-R & GAGCACGAGATGGAGAGTGG \\
\hline
\end{tabular}

